\title{
Técnica de distracción audiovisual para el control de la ansiedad en niño
}

\author{
Audiovisual distraction technique \\ for the control of anxiety in children
}

Valenzuela Muñoz A*, Valenzuela Ramos $M R * *$, Valenzuela Ramos $R^{* * *}$

\section{RESUMEN}

Objetivo. El objetivo del estudio es evaluar la ansiedad del niño antes y después del tratamiento usando la técnica de distracción auditiva visual en niños.

Metodología. Se trata de un estudio cuantitativo, descriptivo y transversal. El estudio fue realizado entre el 2016 y 2017 en dos clínicas estomatológicas pediátricas. Donde se evaluó la ansiedad antes y después del tratamiento utilizando la técnica audiovisual.

Resultados. Se verifica que la ansiedad leve aumenta después de realizado el tratamiento de un $18 \%$ a un $47 \%$. La ansiedad moderada a severa disminuye de un $16 \%$ a un $3 \%$, se observa que ningún niño después de realizado el tratamiento tiene ansiedad severa. Siendo estos resultados estadísticamente significativos. Conclusiones. En conclusión, la técnica de distracción auditiva visual puede ser un método efectivo para reducir los niveles de ansiedad en los pacientes pediátricos durante un tratamiento dental invasivo.

PALABRAS ClAVE: Recursos Audiovisuales, Ansiedad, Niños (DeCs).

\section{ABSTRACT}

Objective. The objective of the study is to assess the child's anxiety before and after treatment using the technique of visual auditory distraction in children.

Methodology. It is a quantitative, descriptive and transversal study. The study was conducted between 2016 and 2017 in two pediatric stomatology clinics. Where anxiety was evaluated before and after treatment using the audiovisual technique.

Results. It is verified that the mild anxiety increases after the treatment is carried out, from $18 \%$ to $47 \%$. Moderate to severe anxiety decreases from $16 \%$ to $3 \%$, it is observed that no child after treatment has severe anxiety. These results being statistically significant.

Conclusions. In conclusion, the technique of visual auditory distraction can be an effective method to reduce the levels of anxiety in pediatric patients during an invasive dental treatment.

KEY WORDS: Audiovisual Aids, Anxiety, Child (DeCs).

Fecha de recepción: 27 de enerol de 2019.

Fecha de aceptación: 2 de febrero de 2019.

Valenzuela Muñoz A, Valenzuela Ramos MR. Valenzuela Ramos R. Técnica de distracción audiovisual para el control de la ansiedad en niño. 2019; 35, (1): 27-31.

* $\quad$ Doctor en Economía. Vicerrector de Investigación Universidad Nacional de Tayacaja. Huancavelica - Perú.

** Doctor en Estomatología. Universidad Señor de Sipán. Lambayeque - Perú.

*** Cirujano Dentista. Servicio de Odontología del Hospital Regional de Lambayeque - Perú. 


\section{INTRODUCCIÓN}

El control del dolor, miedo y ansiedad de los pacientes ante un tratamiento dental siempre ha sido unas de las partes esenciales en la odontología (1). Siendo la ansiedad dental un estado emocional negativo, excesivo e irracional experimentado por los pacientes que acuden a la consulta dental y que se presentan con mayor frecuencia en niños (2).Que lleva a catalogarlo como un problema para el manejo del comportamiento del niño con una prevalencia de 3 a $43 \%$ en diferentes poblaciones (3). La duración del tratamiento dental y el manejo del comportamiento son dos puntos importantes a tener en cuenta cuando se trata de pacientes infantiles. Por lo que se ha sugerido que las citas sean cortas, de lo contrario, los niños interpretarían las sesiones de tratamiento como un signo de problemas importantes que causaría una ansiedad significativa y por ende problemas en el manejo del comportamiento ${ }^{(4)(5)}$.

Existen diferentes técnicas básicas para disminuir la ansiedad dental en niños durante el tratamiento dental, como el programa de entrevistas, el refuerzo positivo, la comunicación no verbal, el control de la voz y la distracción ${ }^{(6)}{ }^{(7)}$. La distracción es una técnica común en el tratamiento dental que distrae la atención del niño de lo que él puede percibir como un tratamiento desagradable ${ }^{(8)}(9)$. Dentro de la técnica de distracción hay dos métodos utilizados en odontología el audio y la distracción auditiva visual. La distracción del audio incluye música, presentación del audio y narración de cuentos a través de auriculares. La distracción auditiva visual incluye la presentación de historias en televisión, realidad virtual y gafas de video tridimensional. La distracción es una técnica simple que no interfiere en la comunicación del niño con el dentista ${ }^{(10)}$.

Aunque hay varios estudio sobre esta técnica relacionadas con el tratamiento dental en niños, estos son controvertidos, con resultados heterogéneos y han sido estudiados en el continente europeo, pero no se han reportado estudios en el continente americano y africano ${ }^{(10)}$. En este sentido, Barreiros ${ }^{(10)}$ recomienda realizar estudios en otras poblaciones con el fin de establecer la efectividad de técnica de distracción auditiva visual en niños en otros antecedentes culturales.

Por lo tanto, el objetivo de la presente investigación es evaluar el nivel de ansiedad del niño antes y des- pués del tratamiento usando la técnica de distracción auditiva visual en niños.

\section{MATERIAL Y MÉTODOS}

Se trata de un estudio cuantitativo, descriptivo y transversal. El estudio fue realizado entre el 2016 y 2017 en dos clínicas estomatológicas pediátricas. El estudio conto con una población de 1161 niños de los cuales fueron seleccionados un total de 400 niños, 207 de sexo masculino y 193 de sexo femenino, de 6 a 8 años de edad.

Se seleccionaron niños que no padecían de alguna enfermedad sistémica, y cuyo comportamiento era levemente negativo, es decir, aquellos que rechazaban el tratamiento, realizaban movimientos leves de las extremidades, tenían un comportamiento tímido y por lo tanto bloqueaban la comunicación, niños que aceptaban y acataban algunas órdenes y cuyo llanto era monotómico según Frankl ${ }^{(11)}$.

A todos los niños se les aplicó la técnica de distracción audiovisual ${ }^{(12)}$ que consistía en presentarles videos narrativos a través de unas gafas de realidad virtual. Esta técnica se realizó durante la ejecución de tratamientos pulpares (pulpotomías y pulpectomías), siendo calificados como tratamientos invasivos, ya que implican el uso de anestesia infiltrativa.

El nivel de ansiedad se midió con la Escala de Imagen Facial (FIS) ${ }^{(13)}$ que tiene que ver con lo cognitivo (sentimiento), donde se le pregunto a cada niños como se sentía antes y después del tratamiento y este debería responder señalando la figura con la que más se sentía identificado, luego se anotó lo manifestado por el niño como ansiedad leve, ansiedad leve a moderada, moderada, ansiedad moderada a severa y ansiedad severa.

\section{RESULTADOS}

Se verifica que la ansiedad leve aumenta después de realizado el tratamiento de un $18 \%$ a un $47 \%$. La ansiedad moderada a severa disminuye de un $16 \%$ a un $3 \%$, se observa que ningún niño después de realizado el tratamiento tiene ansiedad severa. Siendo estos resultados estadísticamente significativos.

En el sexo masculino se verifica un aumento de la ansiedad del $14 \%$ al $44 \%$. La ansiedad leve a 
Tabla 1: Nivel de ansiedad en niños antes y después del tratamiento

\begin{tabular}{lccccc}
\hline \multicolumn{1}{c}{ Nivel de ansiedad } & \multicolumn{2}{c}{ Antes del tratamiento } & \multicolumn{2}{c}{ Después del tatamiento } & Selva \\
Ansiedad leve & 72 & $18 \%$ & 188 & $47 \%$ & $0.0000^{* *}$ \\
Ansiedad leve a moderada & 144 & $36 \%$ & 128 & $32 \%$ & $0.1162 \mathrm{~ns}$ \\
Ansiedad moderada & 80 & $20 \%$ & 72 & $18 \%$ & $0.2355 \mathrm{~ns}$ \\
Ansiedad de moderada a severa & 64 & $16 \%$ & 12 & $3 \%$ & $0.0000^{* *}$ \\
Ansiedad severa & 40 & $10 \%$ & 0 & $0.0000^{* *}$ \\
Muestra & 400 & $\mathbf{1 0 0 \%}$ & $\mathbf{4 0 0}$ & $\mathbf{1 0 0 \%}$ & \\
\hline
\end{tabular}

Significativo ** $\mathrm{p}<0.01 /$ ns no significativo.

Tabla 2: Nivel de ansiedad en niños antes y después del tratamiento según el sexo

\begin{tabular}{|c|c|c|c|c|c|c|c|c|c|c|}
\hline \multirow{3}{*}{$\begin{array}{l}\text { Nivel de ansiedad } \\
\text { Ansiedad leve }\end{array}$} & \multicolumn{5}{|c|}{ MASCULINO } & \multicolumn{5}{|c|}{ FEMENINO } \\
\hline & \multicolumn{2}{|c|}{ Antes del tratamiento } & \multicolumn{2}{|c|}{ Después del tatamiento } & \multirow{2}{*}{$\frac{P}{0.0000 * *}$} & \multicolumn{2}{|c|}{ Antes del tratamiento } & \multicolumn{2}{|c|}{ Después del tatamiento } & \multirow{2}{*}{$\begin{array}{c}P \\
0.0000 * *\end{array}$} \\
\hline & 29 & $14 \%$ & 92 & $44 \%$ & & 45 & $23 \%$ & 99 & $51 \%$ & \\
\hline $\begin{array}{l}\text { Ansiedad leve } \\
\text { a moderada }\end{array}$ & 87 & $42 \%$ & 61 & $30 \%$ & $0.0038 * *$ & 54 & $28 \%$ & 67 & $35 \%$ & $0.9231 \mathrm{~ns}$ \\
\hline $\begin{array}{l}\text { Ansiedad } \\
\text { moderada }\end{array}$ & 29 & $14 \%$ & 50 & $24 \%$ & $0.0043 * *$ & 54 & $28 \%$ & 18 & $9 \%$ & $0.0000 * *$ \\
\hline $\begin{array}{l}\text { Ansiedad de mo- } \\
\text { derada a severa }\end{array}$ & 40 & $19 \%$ & 4 & $2 \%$ & $0.0000 * *$ & 22 & $12 \%$ & 9 & $5 \%$ & $0.0075 \mathrm{~ns}$ \\
\hline Ansiedad severa & 22 & $11 \%$ & 0 & $0 \%$ & $0.0000 * *$ & 18 & $9 \%$ & 0 & $0 \%$ & $0.0000 * *$ \\
\hline Total & 207 & $100 \%$ & 207 & $100 \%$ & & 193 & $100 \%$ & 193 & $100 \%$ & \\
\hline
\end{tabular}

Significativo ${ }^{*} \mathrm{p}<0.01 /$ ns no significativo.

moderada disminuyó de un $42 \%$ a un $30 \%$. La ansiedad moderada aumentó de un $14 \%$ a un $24 \%$. La ansiedad moderada a severa disminuyó de un $19 \%$ a un $2 \%$ y la ansiedad severa disminuyó de un $11 \%$ a un $0 \%$.

En el sexo femenino se aprecia un aumento de un $23 \%$ a un $51 \%$. La ansiedad moderada disminuyó de un $28 \%$ a un $9 \%$. La ansiedad moderada a severa tuvo una disminución de $12 \%$ a un $5 \%$ y en la severa hubo una disminución de un $9 \%$ a un $0 \%$

\section{DISCUSIÓN}

Al evaluar el efecto de la técnica de distracción auditiva visual en niños con diferentes niveles de ansiedad, se demuestra que la ansiedad leve aumenta, la ansiedad moderada a severa y la ansiedad severa disminuyen significativamente después del tratamiento lo que demuestra que la técnica de distracción auditiva visual disminuye la ansiedad en niños, como lo han demostrado varios estudios demostrando las actitudes positivas de los pacientes pediátricos ${ }^{(10)(14)}$.

Además, existen estudios como los realizados por $\mathrm{Al}$ - Khotani ${ }^{(15)}$ y Prabhakar ${ }^{(16)}$ en los cuales se añadieron distintas escalas para evaluar el nivel de ansiedad, tales como la prueba de imagen de Venham, la calificación de Venham de la ansie- dad clínica, conducta cooperativa (MVARS), la frecuencia del pulso y la saturación de oxígeno, a parte de la Escala de Imagen Facial (FIS), mostrando resultados similares al presente estudio. A estos se añade el realizado por Mitrakul (21) que evaluó la ansiedad en las distintas etapas del procedimiento dental.

Sin embargo, Ram ${ }^{(17)}$ no encontró una disminución considerable de la ansiedad utilizando esta técnica, pero si encontró que al compararla con el óxido nitroso ambas técnicas tenían una efectividad similar. Se ha comprobado que la inhalación del óxido nitroso/oxigeno disminuye la ansiedad $^{(18)}$ por lo que se puede explicar los resultados obtenidos en dicho estudio. Agarwal (22) comparo la eficacia de dos anestésicos tópicos en el control del dolor y la ansiedad en niños durante la aplicación de la anestesia dental, empleando la ayuda audiovisual, obteniendo resultados similares al presente estudio.

La duración y la complejidad del tratamiento dental son factores que influyen directamente sobre el nivel de ansiedad en los pacientes pediátricos; para el presente estudio se realizaron tratamientos invasivos y de (pulpotomías y pulpectomías), en los cuales se presentó un aumento de la ansiedad leve en los pacientes, como los encontrados en los estudios de Aminabadi ${ }^{(4)}$ y Jamali ${ }^{(5)}$. Otro es- 
tudio, como el realizado por Hanif ${ }^{(20)}$, indica que además de los factores antes mencionados, el nivel socioeconómico y las visitas dentales previas influyen en el comportamiento y la ansiedad en estos pacientes.

Para formar parte del estudio fueron seleccionados niños de entre 6 a 8 años de edad ya que estos presentan por lo general un comportamiento calificado según Frankl como levemente negativo que son difíciles de controlar. A ello, debe sumarse que a esta edad los niños son lo suficientemente maduros para interactuar con la técnica de distracción auditiva visual durante los procedimientos dentales. Aminabadi ${ }^{(19)}$, sostiene que las técnicas de manejo de comportamiento deberían aplicarse dependiendo de la edad, de las características cognitivas y patrones de comportamiento.

Debido a que existen pocos estudios similares realizados en América Latina, se está frente a un estudio innovador que será de apoyo importante para futuras investigaciones en el área de Odontopediatría de la región en mejora de la calidad de la atención de pacientes ansiosos.

\section{CONCLUSIONES}

En conclusión, la técnica de distracción auditiva visual puede ser un método efectivo para reducir los niveles de ansiedad en los pacientes pediátricos durante un tratamiento dental invasivo.

\section{BIBLIOGRAFIA}

1. Angelo Z, Polyvios C. Alternative practices of achieving anaesthesia for dental procedures: a review. J Dent Anesth Pain Med. abril de 2018;18(2):79-88.

2. Pop-Jordanova N, Sarakinova O, Pop-Stefanova-Trposka M, Zabokova-Bilbilova E, Kostadinovska E. Anxiety, Stress and Coping Patterns in Children in Dental Settings. Open Access Maced J Med Sci. 10 de abril de 2018;6(4):692-7.

3. Lee C-Y, Chang Y-Y, Huang S-T. Prevalence of dental anxiety among 5- to 8-year-old Taiwanese children. J Public Health Dent. 2007;67(1):36-41.
4. Aminabadi NA, Oskouei SG, Farahani RMZ. Dental treatment duration as an indicator of the behavior of 3-to 9-year-old pediatric patients in clinical dental settings. J Contemp Dent Pract. 1 de septiembre de 2009;10(5):E025-032.

5. Jamali Z, Najafpour E, Ebrahim Adhami Z, Sighari Deljavan A, Aminabadi NA, Shirazi S. Does the length of dental procedure influence children's behavior during and after treatment? A systematic review and critical appraisal. J Dent Res Dent Clin Dent Prospects. 2018;12(1):68-76.

6. Adair SM, Rockman RA, Schafer TE, Waller JL. Survey of behavior management teaching in pediatric dentistry advanced education programs. Pediatr Dent. abril de 2004;26(2):151-8.

7. Buchanan H, Niven N. Self-report treatment techniques used by dentists to treat dentally anxious children: a preliminary investigation. Int J Paediatr Dent. enero de 2003;13(1):9-12.

8. Clinical Affairs Committee-Behavior Management Subcommittee, American Academy of Pediatric Dentistry. Guideline on Behavior Guidance for the Pediatric Dental Patient. Pediatr Dent. octubre de 2015;37(5):57-70.

9. Brignardello-Petersen R. Audiovisual distraction resulted in less operator stress than behavior management techniques in cooperative children with special health care needs at the dental office, but there was no improvement in behavior, pain, or appointment time. J Am Dent Assoc. 1 de octubre de 2017;148(10):e138.

10. Barreiros D, Oliveira DSB de, Queiroz AM de, Silva RAB da, Paula-Silva FWG de, Küchler EC. Audiovisual distraction methods for anxiety in children during dental treatment: A systematic review and meta-analysis. J Indian Soc Pedod Prev Dent. 1 de enero de 2018;36(1):2.

11. al TA et. A Study of the Relationship of Parenting Styles, Child Temperament, and Operatory Behavior in Healthy Children. - PubMed NCBI [Internet]. [citado 16 de mayo de 2018]. Disponible en: https://www.ncbi.nlm.nih.gov/ pubmed/29750619

12. Blumer S, Khoury RS, Peretz B. The Prevalen- 
ce of ADHD Patients among Pediatric Dentists in Israel and Knowledge of Dental and Behavioral Aspects of Treating Them. J Clin Pediatr Dent. 2018;42(3):212-6.

13. Buchanan H, Niven N. Validation of a Facial Image Scale to assess child dental anxiety. Int J Paediatr Dent. enero de 2002;12(1):47-52.

14. Zhang C, Qin D, Shen L, Ji P, Wang J. Does audiovisual distraction reduce dental anxiety in children under local anesthesia? A systematic review and meta-analysis. Oral Dis. 2 de marzo de 2018;

15. Al-Khotani A, Bello LA, Christidis N. Effects of audiovisual distraction on children's behaviour during dental treatment: a randomized controlled clinical trial. Acta Odontol Scand. 17 de agosto de 2016;74(6):494-501.

16. Prabhakar AR, Marwah N, Raju OS. A comparison between audio and audiovisual distraction techniques in managing anxious pediatric dental patients. J Indian Soc Pedod Prev Dent. 10 de enero de 2007;25(4):177.

17. Ram D, Shapira J, Holan G, Magora F, Cohen S, Davidovich E. Audiovisual video eyeglass distraction during dental treatment in children. Quintessence Int Berl Ger 1985. septiembre de 2010;41(8):673-9.

18. Guideline on Use of Nitrous Oxide for Pediatric Dental Patients. Pediatr Dent. octubre de
2016;38(6):211-5.

19. Asl Aminabadi N, Erfanparast L, Sohrabi A, Ghertasi Oskouei S, Naghili A. The Impact of Virtual Reality Distraction on Pain and Anxiety during Dental Treatment in 4-6 Year-Old Children: a Randomized Controlled Clinical Trial. J Dent Res Dent Clin Dent Prospects. 2012;6(4):117-24

20. Hanif Raja G, Shafiq Malik F, Bashir U. Dental Anxiety among children of age between 5 to 10 years visiting a teaching dental hospital in Islamabad. Pakistan. J Ayub Med Coll Abbottabad 2015;27(3):587-90.

21. Mitrakul K, Asvanund Y, Arunakul M, Paka-Akekaphat S. Effect of audiovisual eyeglasses during dental treatment in 5-8 yearold children. Eur J Paediatr Dent. 2015; 16 (3): 239-45.

22. Agarwal N, Dhawan J, Kumar D, Anand A, Tangri K. Effectiveness of Two Topical Anaesthetic Agents used along with Audio Visual Aids in Paediatric Dental Patients. J Clin Diagn Res. 2017; 11 (1):80

\section{AUTOR DE CORRESPONDENCIA:}

Marisel Roxana Valenzuela Ramos

Correo electrónico:

mvalenzuela@crece.uss.edu.pe Universidad

Señor de Sipán

Teléfono móvil: 00511956291247 